\title{
Delivery of dCas9 CRISPR System Into the Hard Transfection Cells by Magnetofection Approach
}

\section{Mahdi Mohammadi Ghanbarlou}

National Cell Bank of Iran, Pasteur Institute of Iran

Shahriyar Abdoli

National Cell Bank of Iran, Pasteur Institute of Iran

Hadi Bamehr

National Cell Bank of Iran, Pasteur Institute of Iran

Leila Qazizadeh

National Cell Bank of Iran, Pasteur Institute of Iran

Hamed Omid

National Cell Bank of Iran, Pasteur Institute of Iran

Hamidreza Iranpour

Pasteur Institute of Iran

Hosein Shahsavarani

Pasteur Institute of Iran

Morteza Karimipour

Pasteur Institute of Iran

Mohammad Ali Shokrgozar ( $\square$ mashokrgozar@pasteur.ac.ir)

National Cell Bank of Iran, Pasteur Institute of Iran

\section{Research Article}

Keywords: CRISPR-Cas9, Magnetic Nano Particle, Delivery, Superparamagnetic iron oxide nanoparticles,

Fibroblast

Posted Date: October 28th, 2021

DOl: https://doi.org/10.21203/rs.3.rs-999842/v1

License: (c) (i) This work is licensed under a Creative Commons Attribution 4.0 International License.

Read Full License 


\title{
Delivery of dCas9 CRISPR system into the hard transfection cells by magnetofection approach
}

\section{Evaluating magnetic nanoparticles and Lipofectamine Approaches for delivery of dCas9 CRISPR system into HEK-293 and HFF cells}

\author{
Mahdi Mohammadi Ghanbarlou ${ }^{1,2}$, Shahriyar Abdoli ${ }^{1,3}$, Hadi Bamehr ${ }^{1}$, Leila \\ Qazizadeh $^{1}$, Hamed Omid ${ }^{1}$, Hamidreza Iranpour ${ }^{2}$, Hosein Shahsavarani ${ }^{2,4}$, Morteza \\ Karimipour *5, Mohamad Ali Shokrgozar *1
}

1. National Cell Bank of Iran, Pasteur Institute of Iran, Tehran, Iran.

2. Laboratory of Regenerative Medicine and Biomedical Innovations, Pasteur Institute of Iran, Tehran, Iran.

3. Biotechnology Research Center, Golestan University of Medical Sciences, Golestan, Iran. 4. Department of Cell and Molecular Sciences, Faculty of Life science and Biotechnology, Shahid Beheshti University, Tehran, Iran.

5. Molecular Medicine Dept., Biotechnology Research Center, Pasteur Institute of Iran, Tehran, Iran.

Mohammad Ali Shokrgozar:

National Cell Bank of Iran, Pasteur Institute of Iran, No. 69, Pasteur Ave, Tehran 1316943551, Iran. https://orcid.org/0000-0002-9198-4070

E-mail: mashokrgozar@ pasteur.ac.ir

\section{Morteza Karimipour}

Molecular Medicine Dept., Biotechnology Research Center, Pasteur Institute of Iran, Tehran, Iran. https://orcid.org/0000-0002-2406-5963

E-mail: mortezakarimi@ pasteur.ac.ir 


\begin{abstract}
:
Background: The CRISPR-Cas9 system, a powerful tool, has revolutionized genome engineering in eukaryotic cells and living organisms. However, this approach poses unique concerns and limitations when used by conventional transfection methods, including limited packaging size and low delivery efficiency. Here, we aim at assessing the transfection efficiency of DNA encoding for the CRISPRCas9 system by PEI coated Magnetic NanoParticle (MNPs) to improve the delivery of CRISPR/Cas9 constructs into eukaryotic cells.
\end{abstract}

Results: Superparamagnetic iron oxide nanoparticles (SPIONs) coated with polyethylenimine (PEI) and then complexed with pCXLE-dCas9VPH-T2A-GFP-shP53 plasmid DNA. We used HEK-293 (human embryonic kidney) and Human foreskin fibroblasts (HFF) cells to express GFP after transfection to evaluate delivery efficiency with MNPs and Lipofection methods. PEI-coated nanoparticles with magnetic iron oxide core were synthesized by co-precipitation technique resulting in an average size of $\sim 20 \mathrm{~nm}$ in diameter. Characterization of Magnetic Nano Particle (MNPs) revealed that particles have narrow size distribution sufficient colloidal stability. The result showed that the magnetofection method with an efficiency around $85.7 \%$ for HEK-293 and 28.2\% for HFF. Also, transfection efficiency by lipofection method was $83.2 \%$ and $7.89 \%$ for HEK-293 and HFF respectively.

Conclusion: The magnetofection was revealed to be more efficient than classic Lipofectamine transfection as measured by GFP expression. We show that PEI-MNPs enable effective delivery and improved safety of plasmids encoding CRISPR/Cas9 into eukaryote cells.

Keywords: CRISPR-Cas9, Magnetic Nano Particle, Delivery, Superparamagnetic iron oxide nanoparticles, Fibroblast 


\section{Background}

In biomedicine, from 2012 clustered regularly interspaced short palindromic repeats-associated proteins (CRISPR-Cas9) technology has emerged as a promising and highly efficient approach employed for gene editing in various living organisms and contexts. the CRISPR/Cas9 system allows insertions and deletions at a specific genomic locus through DNA double-strand breaks (DSBs), which are repaired using error-prone non-homologous end joining (NHEJ)-mediated mechanisms $(1,2)$. Furthermore, this system represents the most promising strategy in biomedical applications such as diseases pathology, gene function, gene therapy, and generation of transgenic animal models $(3,4)$.

To date, a wide variety of possible delivery methods have been developed for delivery of CRISPR/Cas9 machinery as form of a ribonucleotide protein (RNP) complex, DNA, viral vector, or mRNA which can be cross cellular barriers of target cells (5). CRISPR/Cas9 delivery systems can be classified into three general types including: 1-physical delivery such as microinjection and electroporation are useful for in vitro delivery (6). Microinjection has been defined as gold standard of CRISPR delivery system by Yang et al 2013 (7). Also, it's no limited to molecular weight of CRISPR/Cas9 component (6). This approach faces critical challenges for in vivo applications (8). 2viral vectors known as the most common delivery vectors and wide range of viral delivery systems are available such as retrovirus, adenovirus (types 2 and 5), adeno-associated virus(AAV), herpes virus, pox virus, human foamy virus (HFV), and lentivirus (9). viral vectors have established advantages such as high transduction efficiency and long term transgene expression ,but their application concern with safety issues and limited packing capacities (10) increased risk of unwanted immunogenicity, expensive large scale production , and risk of integrating viral sequences into the host chromosome (11).3- Non-viral vector such as human serum albumin nanoparticles (NPs), lipid nanoparticles, cell-penetrating peptides, and gold nanoparticles. Represent some advantages over previous approaches include: safety, efficiency and customizability (12). NP based delivery systems can be engineered to target the interest cell or tissues. Also, this approach represents both higher packaging capacity for CRISPR/Cas9 components and protective effect for the loaded cargo against degradation until to reach the host cell. NPs generally are coast effective for scale-up production and very acceptable safety profiles, improved colloidal stability, biocompatibility and low risk of mutagenicity in contrast to viral vectors (13). Despite its advantages, non-viral delivery systems application has been limited due to Low delivery efficiency therefor investigation for development of new polymer or material with optimal delivery efficiency are needed (5). Magnetic nanoparticles (MNPs) primarily has found as a bio-magnetic compasses in bacteria, insects, and larger animals (14). Due to their unique properties such as high magnetization values and ability to cross cellular barriers, 
MNPs has received considerable critical attention and it has shed the light the road to develop of new generation of gene delivery tool (15).

Polyethylenimine (PEI) is a stable, easy to handle, cationic polymer Known as an effective transfection reagent (16). PEI acts by forming a positively charged complex with DNA which led to effective condensation of DNA into compact particles and subsequently binding to anionic residues on the target cell surface $(17,18)$ and cross the cell membrane via endocytosis (19). To date, various surface modified nanoparticles such as graphene oxide- polyethylene glycol-PEI cationic arginine gold NPs, CRISPR-PAsp gold NPs , have been previously assessed for delivering CRISPR/Cas9 components into different cell line (20). In this study, we aimed to compare the efficiency of the delivery of dCas9 CRISPR system into human fibroblast and HEK-293 cell line. Two different method were evaluated for each cell. Also, in each case, magnetofection method in presence of magnetic nanoparticles was used for the comparison with lipofection. So, two different methods and cells were tested to investigation of transfection efficiency. Our study provides a foundation for improvement of transfection method selection.

\section{Results}

\section{Physicochemical properties of CRISPR/Cas9-PEI-SPIONs complex}

PEI coated SPIONs were synthesized as outlined in previous section. It's potential to enter the cell and nucleus analyzed by characterization of physicochemical properties. The optical properties of SPIONPEI NPs can be seen in Fig. 2. 


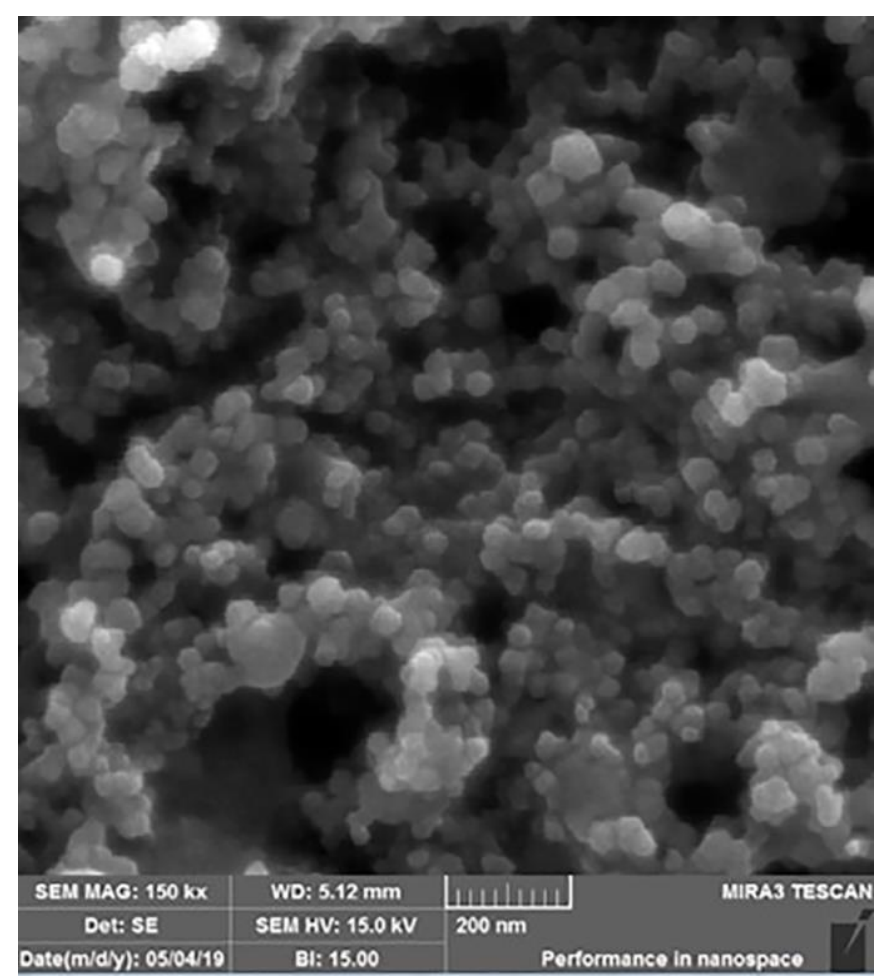

Figure 2. FESEM micrographs of magnetic-nucleic acid complexes

The magnetization curve of $\mathrm{Fe} 3 \mathrm{O} 4$ nanoparticles as shown in fig. 3 reveled that this particles following to synthesis possess superparamagnetic behavior.

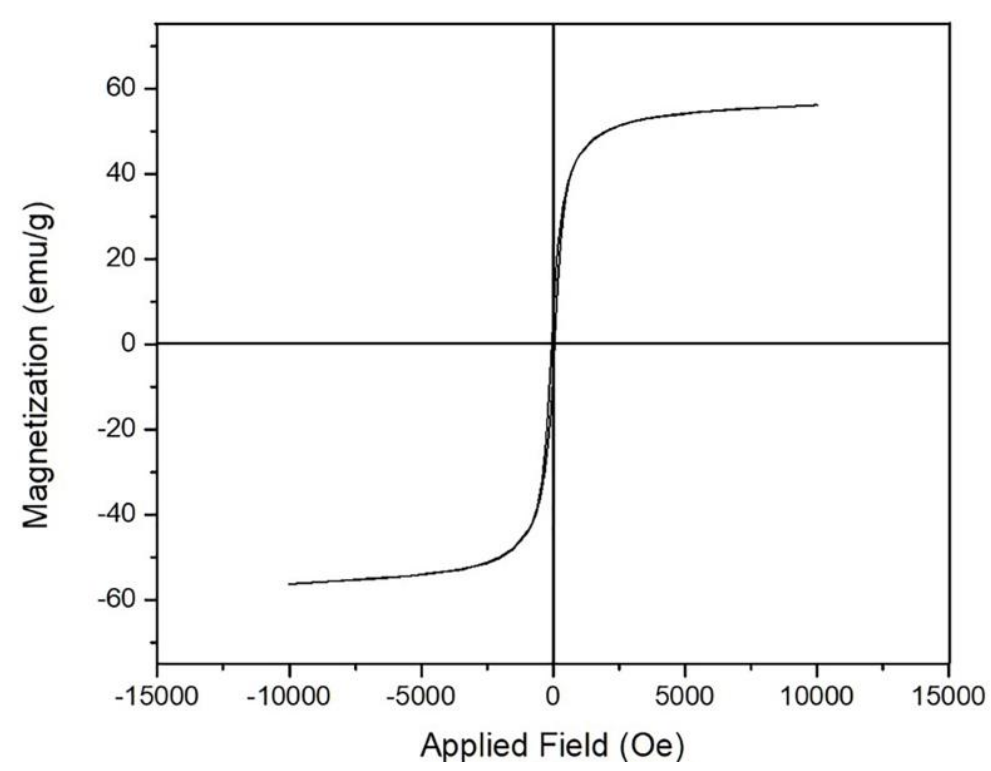

Figure 3. Magnetization curve of SPIONs

It has been observed that the SPION-PEI was well-dispersed and positively charged. Also, the average diameter of SPIONs, SPION-PEI NPs, and CRISPR/Cas9-PEI -SPION particles in the presence of water was around $155 \mathrm{~nm}, 165 \mathrm{~nm}$, and $180 \mathrm{~nm}$ respectively and in the presence of DMEM was 
approximately $265 \mathrm{~nm}, 406 \mathrm{~nm}$, and 800nm as shown in fig. 4 a. Following to surface functionalization of SPIONs by PEI, SPIONs average diameter was slightly increased to $165 \mathrm{~nm}$. The NPs diameter was also increased to $180 \mathrm{~nm}$ after the synthesis of the CRISPR/Cas9-PEI-SPIONs complex. Zeta potential values of SPIONs, SPION-PEI NPs, and CRISPR/Cas9-PEI -SPIONs were 27, 36, $53 \mathrm{mV}$ respectively in water and $-12,-15.5$, and $-16.3 \mathrm{mV}$ in the presence of DMEM supplemented with $10 \% \mathrm{FBS}$ as shown in fig. $4 b$.

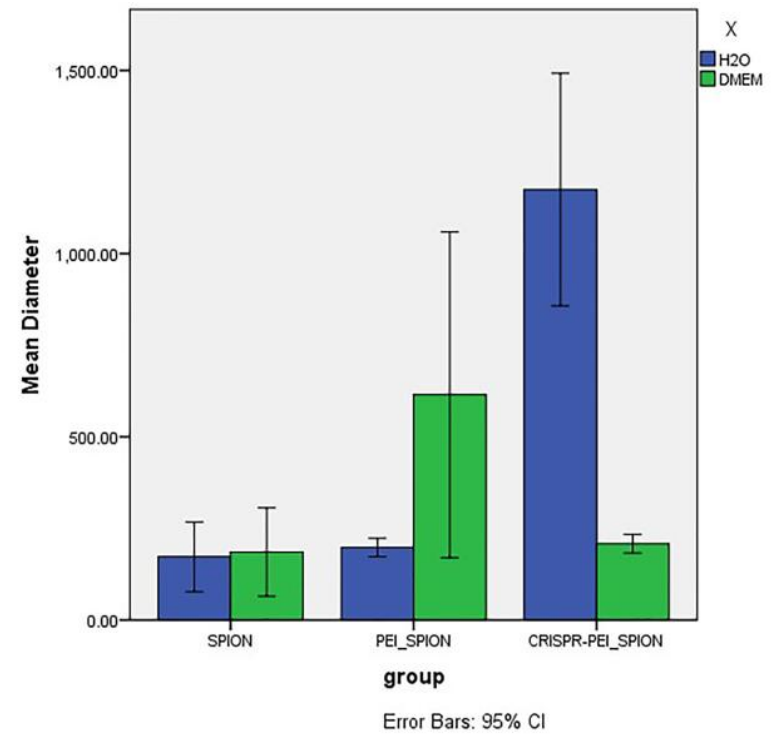

Figure 4a. Hydrodynamic diameter of magnetic nanoparticles (SPIONs), PEI- SPIONs, CRISPR/Cas9-PEISPIONs in the presence of water and DMEM at pH 7.0 (mean \pm S.D.; $\mathrm{n}=3$ ).

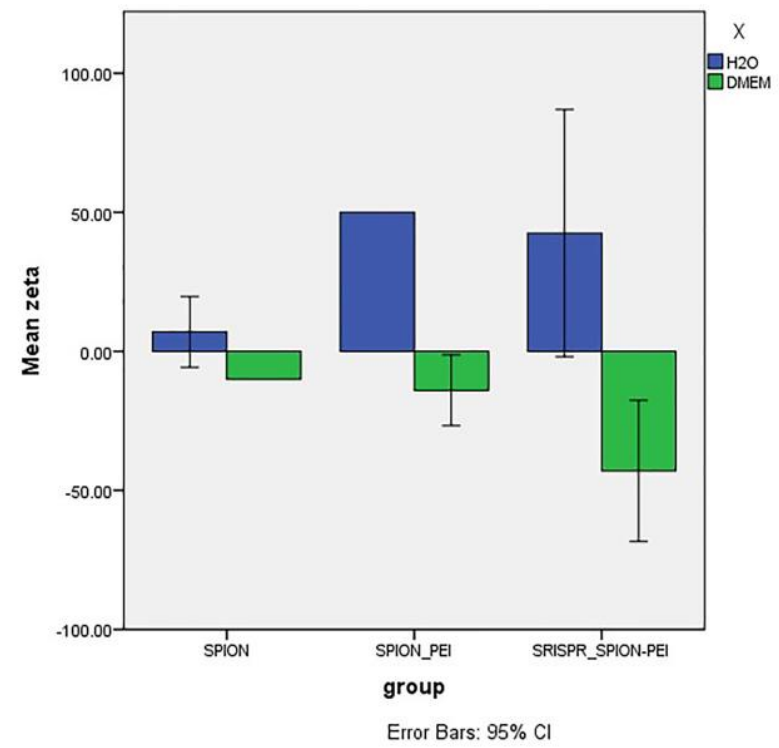

Figure 4b- zeta potential of magnetic nanoparticles (SPIONs), PEI- SPIONs, CRISPR/Cas9-PEI- SPIONs in the presence of water and DMEM at $\mathrm{pH} 7.0($ mean \pm S.D.; $\mathrm{n}=3$ ). 


\section{Fluorescent microscopy}

Fluorescent microscopy images showed that HEK293 and HFF cells morphology 48h post-transfection has no significant differences between magnetofected and lipofected cells. A low number of floating dead cells were observed, as shown in Fig.5.

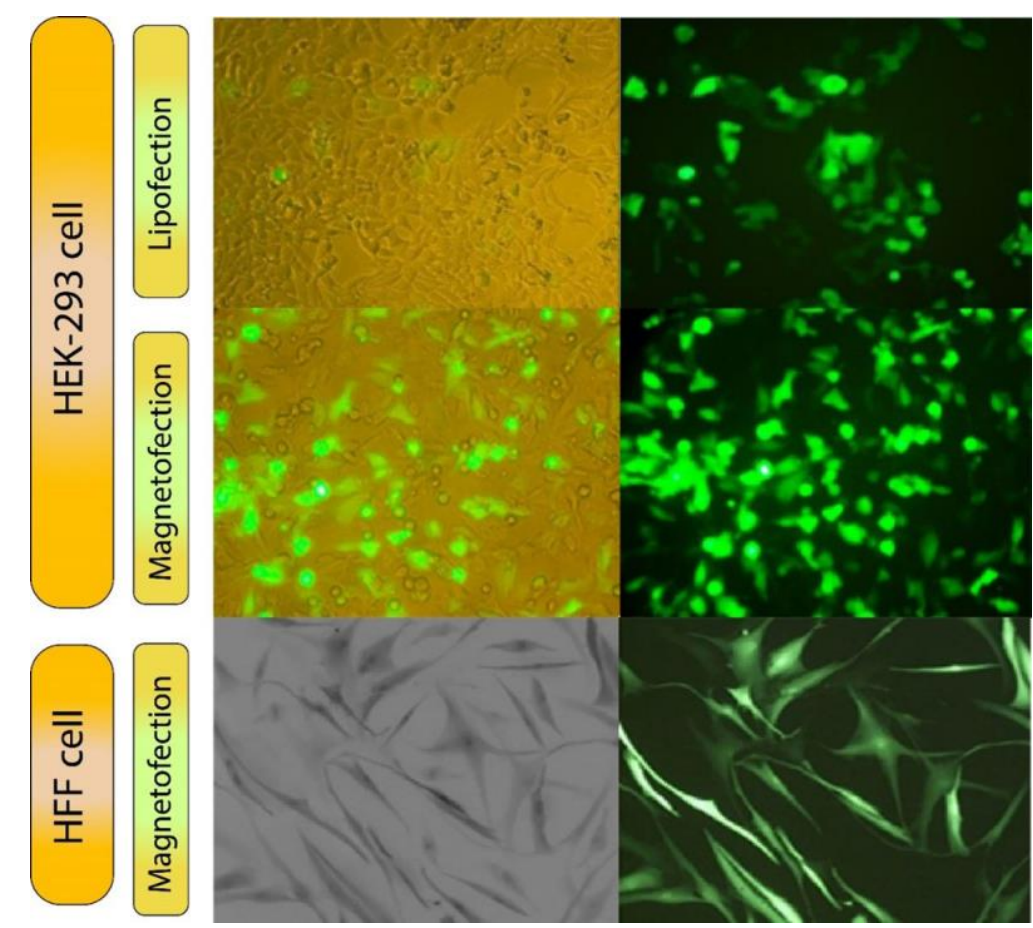

Figure 5. Microscopy images ( $\times 100$ magnification) of non-transfected cells (A), HEK-293 cell s $24 \mathrm{~h}$ after transfection (B), HFF cells $24 \mathrm{~h}$ after transfection

\section{IC50 of CRISPR/Cas9- PEI-SPIONs complex}

To assess the magneto complex's safety for the HEK-293 cell line and HFF cell, the cells were treated with different concentrations of CRISPR/Cas9-PEI-SPION complex, including 5, 10, 20, 40, 60, 80, and DMSO as positive control and then incubated for $24 \mathrm{~h}$. MTT assay showed that IC50 value approximately is $20 \mathrm{pg}$. Therefore; this concentration was used for further analyze.

\section{Cell viability assay}

The survival rate of the HEK-293cell line and HFF cell were measured by MTT (3-(4,5dimethylthiazol-2-yl)-2,5-diphenyltetrazolium bromide) cell toxicity assay. Mean value of viable cells was calculated by determining absorbance at 570/600 $\mathrm{nm}$. Therefore, SPIONs are biocompatible toward HEK-293 and HFF cells. But viability for both transfection methods represent a dose- 
dependent manner and was reduced in magnetofection to $76 \%$; and in lipofection to $82.0 \%$. Also, DMSO was used as a positive control as shown in Figure 6. The result showed that transfection with CRISPR/Cas9- PEI-SPIONS has not significant cytotoxicity associated with the SPIONs compared with the lipofected cell ( $\mathrm{p}$-value $=0.12$ ).

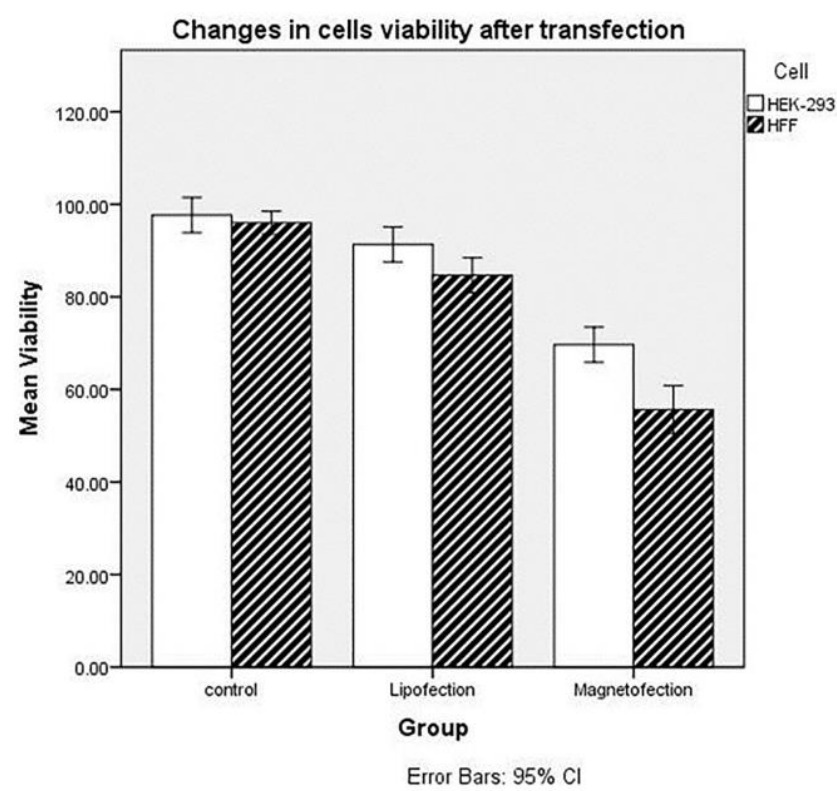

Figure 6. Cellular viability assay. $24 \mathrm{~h}$ post treatment with Lipofectamine or magnetofection SPIONs-MNPs complex

\section{Effect of magnetic field on the magnetofection}

The magnetic fields (SMFs) application to the accumulation of magneto complexes on the cell surface before magnetofection has been investigated and demonstrated to be efficient $(8,21)$. We conducted a transfection experiment with and without a magnetic field to assess whether it can influence the biological pathway such as endocytosis and apoptosis. Also this transfection was done with Lipofectamine. The transfection efficiency was determined as EGF intensity to be around $28.2 \%$ for magnetofection by CRISPR/Cas9-PEI-SPIONs magneto-complexes and 7.89\% for transfection with CRISPR/Cas9-PEI, as shown in Figure 7. 


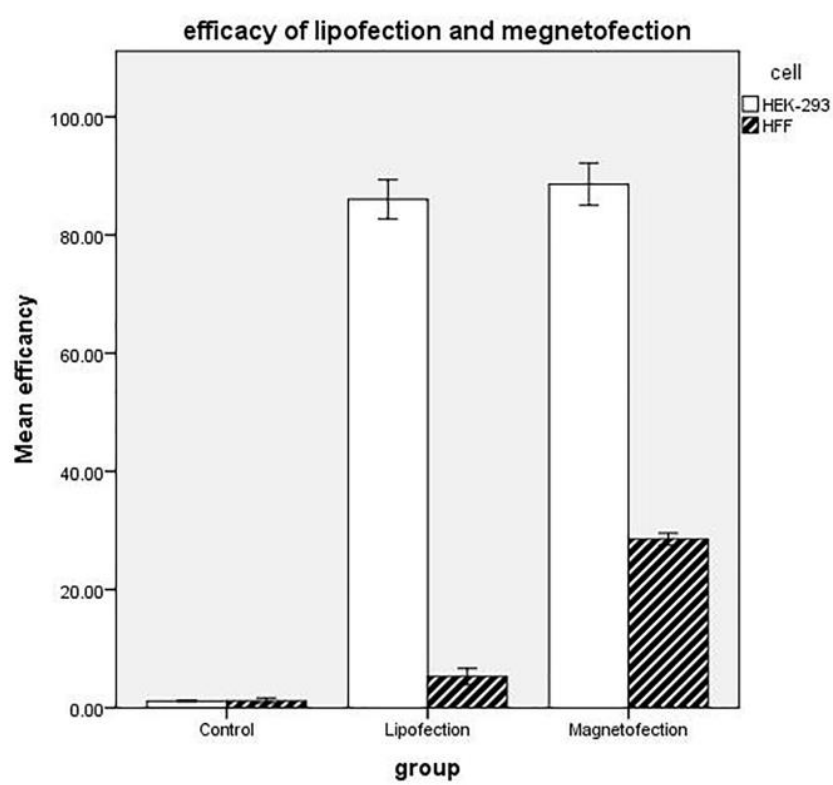

Figure 7. CRISPR/Cas9 genome editing efficacy quantified by GFP intensity in the HEK-293 and HFF. The percentage of transfection efficiency in each tested group was marked on Y-axis.

\section{Cellular uptake of magneto complexes and GFP expression}

To further establish the effect of magneto complexes on cellular uptake, the fluorescence intensity was determined by Flow cytometry experiments. The cellular transfection efficiency measured by Flow cytometry was in line with those obtained by the microscopic observations. The fluorescence intensity of GFP after the Lipofection of CRISPR/Cas9 was observed to be around 7.89, whereas the CRISPR/Cas9-PEI-SPIONs showed transfection efficiency 28.2 for magnetofected cells, as shown in Figure 8. This result proved that Magnetofection has successfully increased the penetration and subsequent dissociation of the CRISPR/Cas9-PEI-SPIONs magneto complexes into the nucleus. 


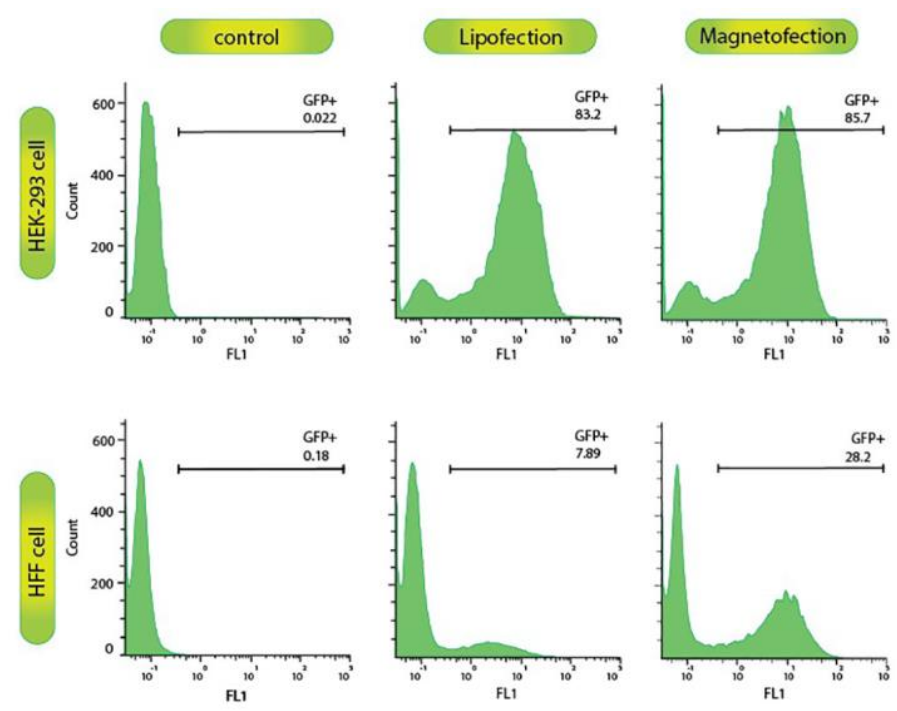

Figure 8. Quantification of GFP intensity results in the HEK-293 and HFF cells using Lipofectamine and CRISPR/Cas9-PEI-SPIONs

\section{Discussion}

CRISPR-Cas9 system, a powerful tool, has revolutionized genome engineering in eukaryotic cells and living organisms. Despite the advantage of Gene delivery through non-viral means, it suffers from low efficacy due to DNA/vector complexes instability in blood circulation and extracellular fluids. The DNA/PEI complexes were also demonstrated to interact by PEI positively charged $\mathrm{NH} 2$ groups to negatively charged heparin sulfate proteoglycans on cells' surfaces (22). Common entry pathway of cargo into a target cell is through clathrin-dependent endocytosis, a size-dependent path $(23,24)$. One advantage of the DNA/PEI complexes is that they represent property such as escaping from endosomes and preventing fusion of endo-lysosome, which are associated with the proton sponge effect $(25,26)$. Therefore it prevents lysis (or burst) of the DNA by DNase or low pH by lysosome (26). MNPs via electrostatic adsorption combined with CRISPR/Cas9-PEI complexes and then transfected into HEK293 and HFF cell line to improve the transfection efficiency. Also, transfection using lipofectamine reagent was conducted, and the result compares to magnetofection. This process is illustrated as Fig 9. 


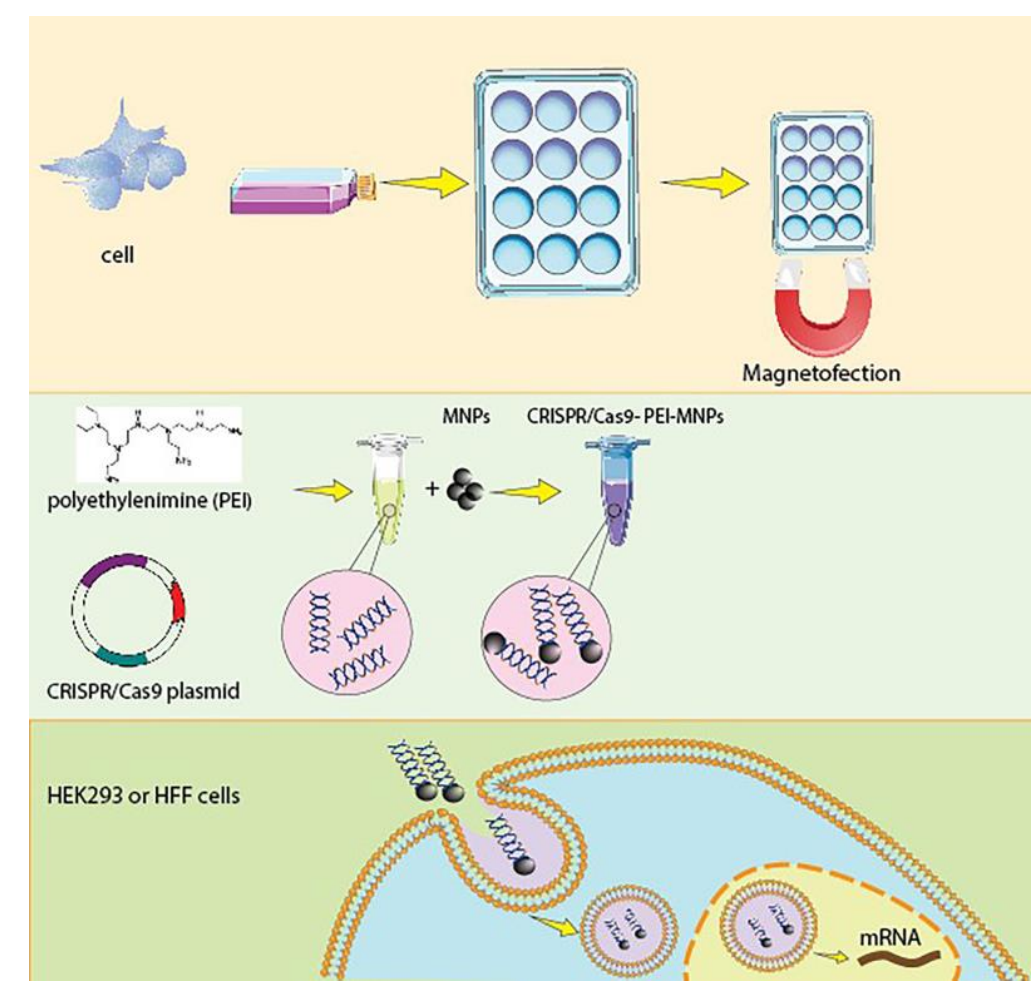

Figure 9. Scheme describing the synthesis of magnetic nanoparticles (MNPs) by co-precipitation method, the fusion of CRISPR/Cas9 plasmids to PEI-MNPs, and magnetofection of this complex into HEK-293 and HFF cells. CRISPR/Cas9- PEI-MNPs cross the cell membrane through the endocytosis pathway.

Our result showed that NPs diameter had been increased following to interaction and formation of CRISPR/Cas9-PEI -SPION. A possible explanation for this might be that PEI contains more hydrophilic groups and result in more interaction with water molecules after suspension. Another possible reason for these results may be the presence of hydrophilic groups of PEI and DNA, which are involved in both association and dissociation of water molecules and caused the formation of a hydration shell around the SPIONs. This result is in line with those conducted by X Wang et al. 2009 (27).

Further, the average diameter of SPIONs, SPION-PEI NPs, and CRISPR/Cas9-PEI -SPION particles in the presence of water and DMEM was different. This finding may be explained by the fact that supplemented DMEM by FBS, Due to serum salt's presence and adsorption onto the surface of PEISPIONs, steric stabilization led to improved vector dispersion in $\operatorname{DMEM}(28)$. Zeta potential values of SPIONs, SPION-PEI NPs, and CRISPR/Cas9-PEI -SPIONs in the presence of water was found to be positively charged. This result may be correlated to the presence of PEI on the outer layer of SPIONs. Moreover, the result showed that Zeta potential values of all NPs configurations in the presence of DMEM supplemented with 10\% FBS became negative. It could be attributed to adsorption of serum components onto the surface of the complex. 
Magneto complex for biological safety investigated with MTT assay. Its result may be explained by the fact that dose-dependent cytotoxicity during magnetofection is correlated to the presence of PEI, which represent the proton sponge effect and cause disruption of endosome/lysosome (17). In this context, Avinash Bajaj et al. reported that transfection efficiency and compatibility of PEI-Cholesterol-Based Lipopolymers is associated with the MW of PEI; they said that PEI-25KDa is highly toxic than other molecular weights of PEI, including 800, 1200, and $2000 \mathrm{KDa}$ (29). Therefore, another possible reason for our result may be the application of commercially available PEI$25 \mathrm{KDa}$. Zahra Sadeghi et al. has evaluated the effect of superparamagnetic iron oxide coated with poly (ethylene glycol)-grafted polyethylenimine (mPEG-co-PEI) for transfection efficiency and reduction of nanoparticles cytotoxicity .they reported that the applied complex, due to the presence of ethylene glycol, was not toxic up to $100 \mu \mathrm{g} / \mathrm{ml}$ in vitro for NT2 cell line (30). Kami et al. have addressed the cytotoxicity of PEI max and PEI max-nanoparticles by Alamar Blue assay, and viability has no differences between groups of mouse embryonic carcinoma cells (CL6) after $48 \mathrm{~h}(31)$ ). in contrast to previous study, Arsianti et al. has reported that transfection of baby hamster kidney cells (BHK2I) with DNA/PEI + MNP, PEI/MNP + DNA/PEI, or PEI/MNP + DNA + PEI vectors has led to significant metabolic activity from $97 \%$ to $60 \%$ (32). A possible explanation for these results may be the lack of adequate experiment setups. Also, further work on the development of a new approach to decrease the cytotoxicity is needed.

Magnetofection in the presence of a magnetic field showed that it could improve the efficiency of delivery. One possible explanation is the electrical potential represented by a magnetic field, which can increase the accumulation and the concentrations of magnetocomplexes on the cell surface. BenediktSteitz colleagues evaluated and showed transfection efficiency of COS cells with superparamagnetic iron oxide nanoparticle coated with polyethylenimine (PEI-SPION) magnetocomplexes with GFP expressing vector was improved (28).

Namgung et al. had investigated the application of PEI-SPION magnetocomplexes to transfection of HEK-293 cells. They reported that CRISPR/Cas9-PEI-spion had entered the HEK-293 cells more efficient the improvement has been considered correlated to enhanced sedimentation of the magnetocomplexes on the HEK-293 cells (21). Hryhorowicz et al. presented that CRISPR/Cas9-PEIMag2 complexed when utilizing the magnetic field's presence can cause enhanced efficacy of transfection .this improved efficacy has been stated to be correlated to enhanced sedimentation of magnetic transfection complexes on the cell surface(33). Lee et al. and colleagues used magnetic duplexes with PEI-modified NPS as generated by Chemical. Cui et al.(34) reported transfection efficacy up to $25 \%$ of Human foreskin fibroblast cells(35). These results in conjugation with fluorescent microscopy provide an insight into the course of events. 
Overall, this result proved that Magnetofection has successfully increased the penetration and subsequent dissociation of the CRISPR/Cas9-PEI-SPIONs magnetocomplexes into the nucleus.

\section{Conclusion}

The CRISPR/Cas9 system has emerged as a promising tool for genome editing of genetic disorders and infectious diseases. Its programmability and ease of use have gained attention, but the efficient delivery CRISPR/Cas9 system remains a significant challenge (35). The use of CRISPR/Cas9-PEIMNPs construct at present of a magnetic gradient field has been demonstrated to be an effective and non-toxic alternative approach in this context, iron oxide like magnetite (Fe3O4) in comparison to other magnetic particle obtained from magnetic transition metals such as Nichol and cobalt present enhanced stability against oxidation and biological compatibility. This study showed that CRISPR/Cas9-PEI-SPIONs to direct delivery of plasmid encoding the GFP could be applied as an optimized transfection method. More research is required to determine the efficacy of SPION MNPs for delivery of a CRISPR/Cas9 genome-editing system for in vivo conditions and local magnetic activation of genome editing.

\section{Materials and Methods}

Chemicals: Ferrous chloride $(\mathrm{FeCl} 2)$, ferrous sulphate $(\mathrm{FeSO} 4.7 \mathrm{H} 2 \mathrm{O})$, sodium hydroxide $(\mathrm{NaOH})$, PEI (PEI; average MW $25 \mathrm{kDa}$, average degree of polymerisation 580), $\mathrm{NaOH}$ were procured from Sigma Aldrich (Deisenhofen, Germany).

\section{Cell culture}

Human foreskin fibroblast cells were isolated from healthy neonatal dooners (age, $\sim 6$ months) at Shariati Hospital, Tehran, Iran. HEK 293 were obtained from National Cell Bank of Iran (Pasteur Institute, Iran). These cell line maintained in Dulbecco's modified Eagle's medium (DMEM; Life Technologies) supplemented with 10\% fetal bovine serum (FBS; Gibco), 2mM GlutaMAX (Life Technologies), and $100 \mu \mathrm{g} / \mathrm{ml}$ penicillin/streptomycin (Life Technologies) and incubated at $37^{\circ} \mathrm{C}$ with $5 \% \mathrm{CO} 2$ incubation. Forty-eight prior to transfection, these cells were seeded at a density of $2 \times 105$ cells per well in 12-well plates (4 plates), separately (TPP, Sigma Aldrich). Plates were incubated at $37^{\circ} \mathrm{C}$ until cells attained a confluency of $\sim 60-70 \%$ at the time of transfection. Two plates were cultured for each cells and two columns of each plates specified as untreated cells or negative control. 
Also, the same plates were cultivated and transfected with CRISPR-Cas9 plasmid DNA with using Lipofectamine 2000 marked as Lipofection group (Invitrogen, Thermofisher Scientific) according to manufacturer's instructions. In the same way, two plate cultivated for magnetofection with CRISPR/Cas9-PEI-MNPs marked as Magnetofection group.

\section{Plasmid}

The dCas9 expression vector, pCXLE-dCas9VP192-T2A-GFP kindly gifted from Timo Otonkoski, Centre of Excellence in Stem Cell Metabolism Helsinki One Health (HOH). The prepared dCas9 construct was propagated in TOP10 cells and then Endo Free Maxi Kit (Qiagen) was implied to purification of transfection-grade pCXLE-dCas9VPH-T2A-GFP-shP53 plasmid DNA. Plasmid without guide RNA generated for negative control purposes. Figurel presents detail of pCXLEdCas9VPH-T2A-GFP-shP53 construct.

\section{Synthesis of Superparamagnetic iron oxide nanoparticles (SPIONs)}

$\mathrm{Fe} 3 \mathrm{O} 4$ magnetic nanoparticles (MNPs) consist of magnetite $(\gamma$-Fe2O3) or magnetite $(\mathrm{Fe} 3 \mathrm{O} 4)$ particles with size ranging from 1 and $100 \mathrm{~nm}$. These particles were generated by an in situ co-precipitation method as reported by Tiwari et al. in 2016 (36). Briefly, FeCl3.6H2O (1.0 mmol) and FeSO4.7H2O $(2.0 \mathrm{mmol})$ were mixed in $100 \mathrm{ml}$ deionized water and then heated to $80{ }^{\circ} \mathrm{C}$. After that, the $\mathrm{NaOH}$ solution $(1.0 \mathrm{M})$ was slowly added to the mixture until the $\mathrm{pH}$ reached up to 11 with shaking to yield the precipitate. The precipitate color turned black from the light brown, which indicating the formation of Fe3O4 NPs. The black mixture was stirred for $15 \mathrm{~min}$, undergone the hydrothermal treatment by transferring it to the sealed autoclave, and aged for $30 \mathrm{~min}$ at $70{ }^{\circ} \mathrm{C}$. Following this; the reaction mixture was cooled down to room temperature. The SPIO nanoparticles were separated by magnetic separation, and the sample impurities were removed by washing with deionized water. Finally, the SPION was suspended in the deionized water for further use.

\section{Synthesis of PEI coated MNPs and complexed with CRISPR-Cas9 plasmid}

Preparation of magnetic complexes was done by conjugating the aqueous solution of PEI and DNA with a nitrogen in PEI-coated MNPs to phosphorus in DNA (N/P) conjugation ratio of 10/1 as reported by Zhang et al. 2014. Briefly, Magnetic complexes were prepared by mixing $50 \mu \mathrm{lof}$ PEI ( $40 \mu \mathrm{g} / \mathrm{mL})$ and $2 \mu \mathrm{g}$ of plasmid DNA, and the mixture was incubated with MNPs for 20 min at room temperature 
(37, 38). After that, the CRISPR/Cas9-PEI-MNPs complex, which has MNPs to CRISPR-Cas9 plasmid weight ratio of 10 used for further experiment.

\section{Physicochemical characterization.}

The size distribution and zeta potential of CRISPR/Cas9-PEI -SPION was determined using the DLS device (Sympatech, NANOPHOX Model, Germany). Samples sonicated for 1-2 minutes in injectable distilled water. He-Ne laser beam measurements were conducted by detecting at a scattering angle $90^{\circ}$ at $633 \mathrm{~nm}$ at $25^{\circ} \mathrm{C}$. The zeta potential determine using a universal zeta dip cell. The morphology and the particle size of the MNPs were analyzed by FESEM microscopy. The magnetic properties of the synthesised $\mathrm{Fe} 3 \mathrm{O} 4$ was evaluated by vibrating sampling magnetometer (VSM) at room temperature.

\section{Magnetofection}

One day before magnetofection/transfection, the cells were seeded at cell density $\left(2 \times 10^{5}\right.$ cells per well, 6-well plate) (TPP, Sigma Aldrich) and grown in DMEM containing 10\% FBS. The degree of cell confluency at the time of transfection was about $\sim 70 \%$. For magnetofection, the CRISPR/Cas9PEI-MNPs (20 pg DNA/ cell) $(2 \mu \mathrm{g}$ DNA/ well) (1000 $\mu$ l were added to each well) were added to cultured cells in serum-free media for $2 \mathrm{~min}$, followed by the addition of $10 \%$ FBS. The plate was placed on a sintered Mega Magnetic Plate and incubated for $20 \mathrm{~min}\left(37^{\circ} \mathrm{C}, 5 \% \mathrm{CO} 2\right)$ to assess the magnetofection potential to help the complex spun the cells surface. This step followed by a $24 \mathrm{~h}$ incubation period with a fresh media in the absence of magnetic field at $37 \circ \mathrm{C}, 5 \% \mathrm{CO} 2$ humidified atmosphere.

\section{Evaluation of transfection efficiency by fluorescent microscopy}

Following transfection, plates were incubated for $24 \mathrm{~h}$ with these magnetic complexes. Cell growth and morphology were assessed for control, lipofection, and magnetofection groups using fluorescence microscopy.

\section{Flow cytometer analyses of fluorescence intensity}

After $24 \mathrm{~h}$ post-transfection, cells were trypsinized, washed three times with a PBS solution to remove the bounded MNPs, finally harvested, and re-suspended in fresh DMEM for further analysis by flow cytometer. Positive GFP-expressing cells were analyzed using a flow cytometer instrument. The presented data were analyzed using FlowJo software V10 software. Fluorescence intensity percent, 
which indicates the transfection efficiency, were determined by forward scatter area (FSC-A), FSC width (FSC-W), and side scatter area (SSC-A). GFP fluorescence intensity was graphed using a dimensional dot plot of $488 \mathrm{~nm}$ excitation and emission detected at 530/30 versus 575/30 $\mathrm{nm}$ or 530/30 versus $695 / 40 \mathrm{~nm}$, respectively.

\section{Toxicity assay}

The cytotoxicity of the magnetic complexes was evaluated using MTT cell toxicity assay. The cells were seeded at a density of $1 \times 10^{3}$ cells/well on a 96-well plate (TPP, Sigma Aldrich) in $100 \mu \mathrm{L}$ of supplemented DMEM and incubated for $24 \mathrm{~h}$ until reached to confluency of $70 \%$. The cells were transfected with 20 pg of CRISPR/Cas9-PEI-SPIONs using Mega Magnetic Plate. Also, other plates transfected with Lipofectamine and then incubated for $24 \mathrm{~h}$. Following that, the supernatant was replaced with fresh DMEM and then $25 \mu \mathrm{L}$ of a $5.5 \mathrm{mg} / \mathrm{mL}$ MTT reagent was added to the cells. The plates were incubated for $3 \mathrm{~h}$ in the dark, and finally, the supernatant was removed and the remaining purple formazan was lysed with DMSO100 $\mu 1 / 1$ for $40 \mathrm{~min}$. The assay was performed in triplicate. Absorbance was measured using ELISA Reader (Bio Tek Instruments, Inc., VT, USA) at 570nm. The cell viability was calculated from the absorbance versus concentration curve.

\section{Statistical analysis}

The experiments were done in triplicate, and The Results are expressed as means \pm standard deviation. A paired t-test, one-way analysis of variance with Bonferroni's post hoc test, or two-way analysis of variance with post hoc test were used for statistical analysis. The P-values of $<0.05$ were considered significant. Statistical analysis was performed using commercially available software IBM $^{\circledR}$ SPSS $^{\circledR}$ Statistics software (IBM Corp., Armonk, NY, USA).

\section{Ethics approval and consent to participate}

All the participants were thoroughly informed about the study and procedures before signing consent forms. Participants were assured of anonymity and confidentiality. The Research Ethics Committee of the Pasteur Institute of Iran, Tehran, approved this study (IR.NIMAD.REC.1398.051).

\section{Consent for publication}

All authors have participated in conception and design, or analysis and interpretation of the data; drafting the article or revising it critically for important intellectual content; and approval of the final version. 


\section{Availability of data and materials}

All data generated or analyzed in this study are included in the present article.

\section{Funding}

Data of this research was obtained from $\mathrm{PhD}$ dissertation of MMG and financially supported by Pasteur Institute of Iran (Thesis No. BD-9473).

\section{Authors' contributions}

MK and MS conceived and designed the study. MMG performed experiments, data analysis and writing initial draft of the manuscript. HI, HB, LG, HO, SA and HS checking data analysis.

\section{Acknowledgements}

This study was supported in part by the Funding Program from postgraduate office, Pasteur Institute of Iran, Tehran, Iran.

\section{References}

1. Song F, Stieger K. Optimizing the DNA donor template for homology-directed repair of double-strand breaks. Molecular Therapy-Nucleic Acids. 2017;7:53-60.

2. Sánchez-Rivera FJ, Jacks T. Applications of the CRISPR-Cas9 system in cancer biology. Nature reviews cancer. 2015;15(7):387.

3. Riordan SM, Heruth DP, Zhang LQ, Ye SQ. Application of CRISPR/Cas9 for biomedical discoveries. Cell \& bioscience. 2015;5(1):1-11.

4. Khadempar S, Familghadakchi S, Motlagh RA, Farahani N, Dashtiahangar M, Rezaei H, et al. CRISPR-Cas9 in genome editing: Its function and medical applications. Journal of cellular physiology. 2019;234(5):5751-61.

5. Li L, He Z-Y, Wei X-W, Gao G-P, Wei Y-Q. Challenges in CRISPR/CAS9 delivery: potential roles of nonviral vectors. Human gene therapy. 2015;26(7):452-62.

6. Lino CA, Harper JC, Carney JP, Timlin JA. Delivering CRISPR: a review of the challenges and approaches. Drug delivery. 2018;25(1):1234-57.

7. Horii T, Arai Y, Yamazaki M, Morita S, Kimura M, Itoh M, et al. Validation of microinjection methods for generating knockout mice by CRISPR/Cas-mediated genome engineering. Scientific reports. 2014;4:4513. 
8. $\quad \mathrm{Li} \mathrm{L}, \mathrm{Hu} \mathrm{S}$, Chen X. Non-viral delivery systems for CRISPR/Cas9-based genome editing: Challenges and opportunities. Biomaterials. 2018;171:207-18.

9. Huang Y, Liu X, Dong L, Liu Z, He X, Liu W. Development of viral vectors for gene therapy for chronic pain. Pain research and treatment. 2011;2011.

10. Yin H, Song C-Q, Dorkin JR, Zhu LJ, Li Y, Wu Q, et al. Therapeutic genome editing by combined viral and non-viral delivery of CRISPR system components in vivo. Nature biotechnology. 2016;34(3):328-33.

11. Xu CL, Ruan MZ, Mahajan VB, Tsang SH. Viral delivery systems for CRISPR. Viruses. 2019;11(1):28.

12. Javadzadeh Y, Bahari LA. Therapeutic nanostructures for dermal and transdermal drug delivery. Nano-and Microscale Drug Delivery Systems: Elsevier; 2017. p. 131-46.

13. Givens BE, Naguib YW, Geary SM, Devor EJ, Salem AK. Nanoparticle-based delivery of CRISPR/Cas9 genome-editing therapeutics. The AAPS journal. 2018;20(6):108.

14. Walker MM, Diebel CE, Haugh CV, Pankhurst PM, Montgomery JC, Green CR. Structure and function of the vertebrate magnetic sense. Nature. 1997;390(6658):371-6.

15. Shubayev VI, Pisanic II TR, Jin S. Magnetic nanoparticles for theragnostics. Advanced drug delivery reviews. 2009;61(6):467-77.

16. Mady MM, Mohammed W, El-Guendy NM, Elsayed AA. Effect of polymer molecular weight on the DNA/PEI polyplexes properties. Rom J Biophys. 2011;21(2):151-65.

17. Tomitaka A, Koshi T, Hatsugai S, Yamada T, Takemura Y. Magnetic characterization of surface-coated magnetic nanoparticles for biomedical application. Journal of Magnetism and Magnetic Materials. 2011;323(10):1398-403.

18. Boussif O, Lezoualc'h F, Zanta MA, Mergny MD, Scherman D, Demeneix B, et al. A versatile vector for gene and oligonucleotide transfer into cells in culture and in vivo: polyethylenimine. Proceedings of the National Academy of Sciences. 1995;92(16):7297-301.

19. Kircheis R, Wightman L, Wagner E. Design and gene delivery activity of modified polyethylenimines. Advanced drug delivery reviews. 2001;53(3):341-58.

20. Rohiwal S, Dvorakova N, Klima J, Vaskovicova M, Senigl F, Slouf M, et al. Polyethylenimine based magnetic nanoparticles mediated non-viral CRISPR/Cas9 system for genome editing. Scientific reports. 2020;10(1):1-12.

21. Namgung R, Singha K, Yu MK, Jon S, Kim YS, Ahn Y, et al. Hybrid superparamagnetic iron oxide nanoparticle-branched polyethylenimine magnetoplexes for gene transfection of vascular endothelial cells. Biomaterials. 2010;31(14):4204-13.

22. Godbey W, Wu KK, Mikos AG. Tracking the intracellular path of poly (ethylenimine)/DNA complexes for gene delivery. Proceedings of the National Academy of Sciences. 1999;96(9):5177-81. 23. Pichon C, Gonçalves C, Midoux P. Histidine-rich peptides and polymers for nucleic acids delivery. Advanced drug delivery reviews. 2001;53(1):75-94.

24. Ota S, Takahashi Y, Tomitaka A, Yamada T, Kami D, Watanabe M, et al. Transfection efficiency influenced by aggregation of DNA/polyethylenimine max/magnetic nanoparticle complexes. Journal of nanoparticle research. 2013;15(5):1653.

25. Freeman EC, Weiland LM, Meng WS. Modeling the proton sponge hypothesis: examining proton sponge effectiveness for enhancing intracellular gene delivery through multiscale modeling. Journal of Biomaterials Science, Polymer Edition. 2013;24(4):398-416.

26. Ma Y, Zhang Z, Wang X, Xia W, Gu H. Insights into the mechanism of magnetofection using MNPs-PEI/pDNA/free PEI magnetofectins. International journal of pharmaceutics. 2011;419(12):247-54.

27. Wang X, Zhou L, Ma Y, Li X, Gu H. Control of aggregate size of polyethyleneimine-coated magnetic nanoparticles for magnetofection. Nano Research. 2009;2(5):365-72.

28. Steitz B, Hofmann H, Kamau SW, Hassa PO, Hottiger MO, von Rechenberg B, et al. Characterization of PEI-coated superparamagnetic iron oxide nanoparticles for transfection: Size 
distribution, colloidal properties and DNA interaction. Journal of Magnetism and Magnetic Materials. 2007;311(1):300-5.

29. Bajaj A, Kondaiah P, Bhattacharya S. Synthesis and gene transfection efficacies of PEIcholesterol-based lipopolymers. Bioconjugate chemistry. 2008;19(8):1640-51.

30. Sadeghi Z, Maleki P, Shahabi F, Bondarkhilli SAM, Masoumi M, Taheri M, et al. Surface modification of superparamagnetic iron oxide (SPION) and comparison of cytotoxicity effect of mPEG2000-PEI-SPION and mPEG750-PEI-SPION on the human embryonic carcinoma stem cell, NTERA2 cell line. Human Antibodies. (Preprint):1-9.

31. Kami D, Takeda S, Makino H, Toyoda M, Itakura Y, Gojo S, et al. Efficient transfection method using deacylated polyethylenimine-coated magnetic nanoparticles. Journal of Artificial Organs. 2011;14(3):215.

32. Arsianti M, Lim M, Marquis CP, Amal R. Assembly of polyethylenimine-based magnetic iron oxide vectors: insights into gene delivery. Langmuir. 2010;26(10):7314-26.

33. Hryhorowicz M, Grześkowiak B, Mazurkiewicz N, Śledziński P, Lipiński D, Słomski R. Improved delivery of CRISPR/Cas9 system using magnetic nanoparticles into porcine fibroblast. Molecular biotechnology. 2019;61(3):173-80.

34. Cui J, Cui H, Wang Y, Sun C, Li K, Ren H, et al. Application of PEI-modified magnetic nanoparticles as gene transfer vector for the genetic modification of animals. Advances in Materials Science and Engineering. 2012;2012.

35. Lee $\mathrm{CH}$, Kim J-H, Lee HJ, Jeon K, Lim H, yeon Choi H, et al. The generation of iPS cells using non-viral magnetic nanoparticlebased transfection. Biomaterials. 2011;32(28):6683-91.

36. Tiwari AP, Rohiwal SS, Suryavanshi MV, Ghosh SJ, Pawar SH. Detection of the genomic DNA of pathogenic $\alpha$-proteobacterium Ochrobactrum anthropi via magnetic DNA enrichment using pH responsive BSA@Fe 3 O 4 nanoparticles prior to in-situ PCR and electrophoretic separation. Microchimica Acta. 2016;183(2):675-81.

37. Wightman L, Kircheis R, Rössler V, Carotta S, Ruzicka R, Kursa M, et al. Different behavior of branched and linear polyethylenimine for gene delivery in vitro and in vivo. The Journal of Gene Medicine: A cross-disciplinary journal for research on the science of gene transfer and its clinical applications. 2001;3(4):362-72.

38. Zhang L, Li Y, Jimmy CY, Chen YY, Chan KM. Assembly of polyethylenimine-functionalized iron oxide nanoparticles as agents for DNA transfection with magnetofection technique. Journal of Materials Chemistry B. 2014;2(45):7936-44. 